\title{
Gaseous Aliphatic Aldehydes in Chinese Incense Smoke
}

\author{
J. M. Lin, L. H. Wang
}

Division of Environmental Health, College of Public Health, National Taiwan University, Room 1451, No. 1 Jen-Ai Road, 1st Section,

Taipei, Taiwan, Republic of China

Received: 13 August 1993/Accepted: 30 January 1994

Aliphatic aldehydes were found during the combustion of materials. Tobacco smoke contains aldehydes (Crupm and Gardiner, 1989). Fire fighters were exposed to aldehydes when they conducted firefighting (Brandt-Rauf et al, 1988). Aldehydes in ambient air come mainly from the incomplete combustion of hydrocarbons and from photochemical reaction (World Health Organization, 1987). Most aldehydes in ambient air are formaldehyde and acetaldehyde (Kalabokas et al, 1988). Formaldehyde, acetaldehyde, propionaldehyde, butyraldehyde, and benzaldehyde were found in the atmosphere in Los Angeles (Grosjean, 1982).

Burning Chinese incense for whorshipping deities is a Chinese daily routine. It was suspected to be a factor causing nasopharyngeal cancer(Mimi et al, 1990; Chen et al 1987). Epidemiological studies correlated it with the high risk of childhood brain tumor (Martin et al, 1982) and the high risk of childhood leukemia (Lowengard et al, 1987). Ames test identified the mutagenic effect of the smoke from burning Chinese incense (Rasmussen, 1987). The smoke had been proved to contain polycyclic aromatic hydrocarbons and aromatic aldehydes (Schoenotol and Gibbard, 1967). Suspicion about formaldehyde and other alphatic aldehydes was evoked, when a survey of indoor air pollution was conducted in Taipei city (Lin and Yao, 1993). This study determined the presence of aliphatic aldehydes in the smoke from burning Chinese incense under a controlled atmosphere.

\section{MATERIALS AND METHODS}

Four types of Chinese incense containing sandalwood powder were used in this study. They were made in Taiwan, Mainland China and Japan. A chamber $\left(68 \times 48 \times 44 \mathrm{~cm}^{3}\right)$ was constructed by using polypropylene, for burning the Chinese stick incense. A constant 
flow $(5 \mathrm{~L} / \mathrm{min})$ of purified air was humidified by applying a FlowTemperature-Humidity Control System (Model HCS 301, Miller Nelson Research) and flowed through the chamber to the exhaust. Temperature and humidity inside the chamber were monitored by thermometer and Dew Point Meter (model 91HC, YSI). A stick of incense was ignited by a nickel-chrome wire controlled by a voltage regulator. The smoke generated in the chamber was collected by a 2,4-dinitrophenyl-hydrazine coated Sep-Pak DNPHsilica cartridge (Part No.37500, Millipore) in $2 \mathrm{~L} / \mathrm{min}$ for $2 \mathrm{~min}$. Then, the Sep-Pak was eluted by $5 \mathrm{~mL}$ acetonitrile (BDH, Hipersolv, England). The high performance liquid chromatograph (Model 590 , Naters, USA) equipped with U.V detector at $\lambda \max 254 \mathrm{~nm}$ was utilized to determine the derivatives of aliphatic aldehydes in the eluent. The injection volume was $10 \mu \mathrm{L}$. The column was Nova-Pak $\mathrm{Cl} 8(150 \mathrm{~mm} \times 3.9 \mathrm{~mm})$ and the flow rate of the solvent system (acetonitrile/ $/ \mathrm{H}_{2} 0 ; 60 / 40 \mathrm{v} / \mathrm{v}$ ) was $1.0 \mathrm{~mL} / \mathrm{min}$. A quantified and mixed aldehydes standard solution obtained from the aldehydes standard solution kit (TK-151, Alltech) was spiked onto the SepPak and eluted by $5 \mathrm{~mL}$ acetonitrile for forming the stock solution of aldehydes 2,4-dinitrophenyl-hydrazones. From this solution, a series of standard solution was prepared. A quantified 4fluorobenzonitrile (46680, Fluka) in acetonitrile $(0.134 \mathrm{~g} / 10 \mathrm{~mL})$ serving as an internal standard was added to the standard solutions and to the eluents of the samples. The retention time of each derivative of aldehydes relative to that of the internal standard was determined. The relative peak area for each aldehyde derivative was also calculated when a sample was quantified. After the analysis by HPLC were completed, the samples were again analyzed by employing GC/MS (gas chromatograph / mass spectrometer, HP 5890 SeriesII/HP 5971) equipped with HPG 1034B HS Chemstation. The column of GC was HP-1 column (methylene silicon $12 \mathrm{~m} \times 0.2 \mathrm{~mm} \times 3 \mu \mathrm{m})$. Helium was used as the carrier gas, at a flow rate of $0.72 \mathrm{~mL} / \mathrm{min}$. The operating conditions of the mass spectrometer were: ionization energy $70 \mathrm{ev}$, accelerating voltage 1055 volt, and resolution 1 amu.

A field sample was also collected by a Sep-Pak DNPH-silica cartridge and analyzed by HPLC. The cartridge was located $150 \mathrm{~cm}$ above ground and $10 \mathrm{~cm}$ away from an incense holder in a courtyard of Lungshan Temple, a Chinese temple in Taipei.

\section{RESULTS AND DISCUSSION}

Figure 1. shows the HPLC chromatograms of the derivatives of aldehydes in the smoke from burning four types of Chinese incense. Formaldehyde, acetaldehyde, acrole in and propionaldehyde were 


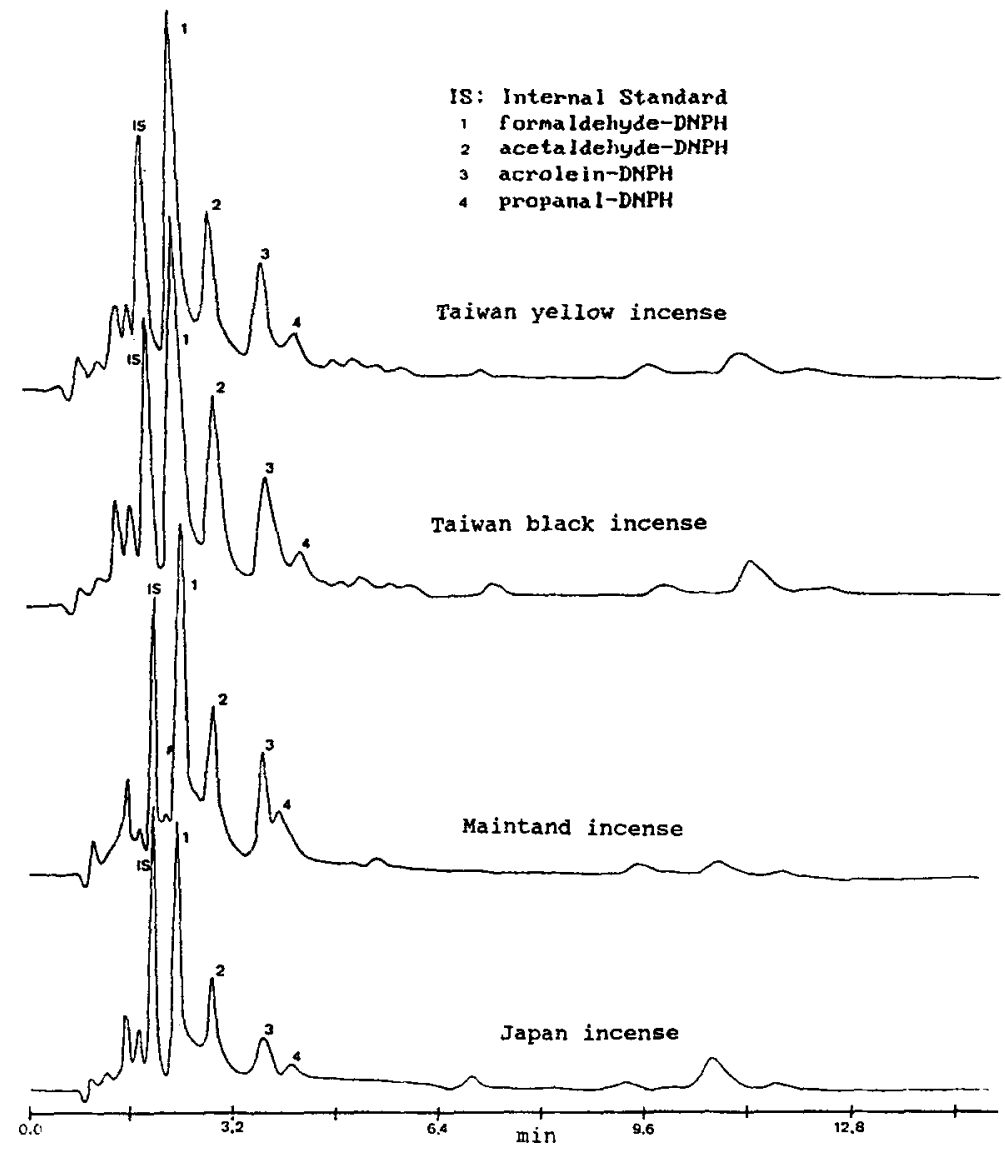

Figure 1. The HPLC chromatograms of the derivatives of aldehydes in the smoke from burning four types of Chinese incense.

Table 1. Retention time of aldehydes-DNPH relative to internal standard

\begin{tabular}{|c|c|c|c|c|}
\hline \multirow[b]{2}{*}{ Aldehydes-DNPH* } & \multicolumn{4}{|c|}{ Relative retention time } \\
\hline & $n * *$ & Mean & S.D. & C.V. ( $(8)$ \\
\hline formaldehyde-DNPH & 12 & 1.217 & 0.007 & 5.75 \\
\hline acetaldehyde-DNPH & 12 & 1.495 & 0.019 & 1.27 \\
\hline acrolein-DNPH & 12 & 1.910 & 0.030 & 1.57 \\
\hline propanal-DNPH & 12 & 2.134 & 0.034 & 1.59 \\
\hline methacrolein-DNPH & 12 & 2.817 & 0.070 & 2.48 \\
\hline butanal-DNPH & 12 & 2.981 & 0.040 & 1.34 \\
\hline
\end{tabular}

identified based on retention times relative to the internal standard as shown in Table 1. 


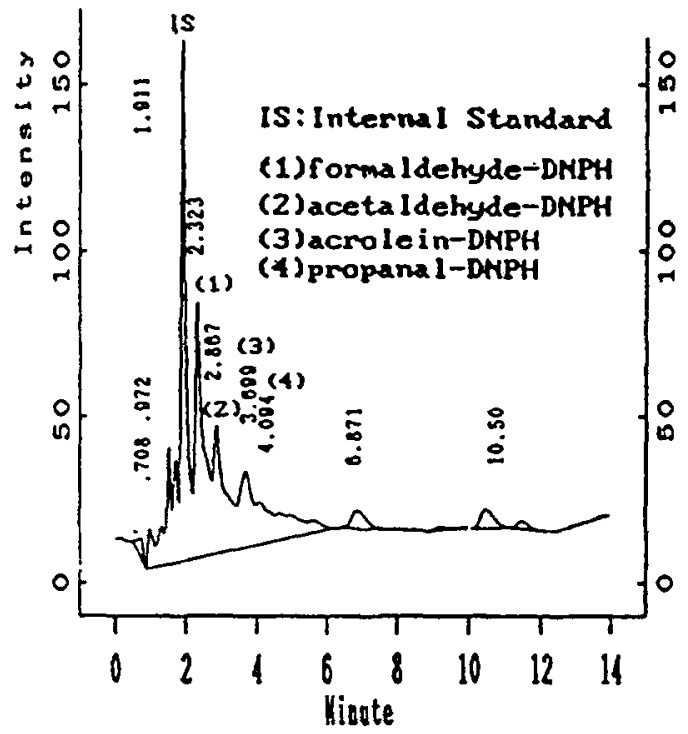

Figure 2. The HPLC chromatogram of the DNPH-derivatives of aldehydes in the smoke collected from a Chinese temple.(DNPH: dini trophenylhydrazone)

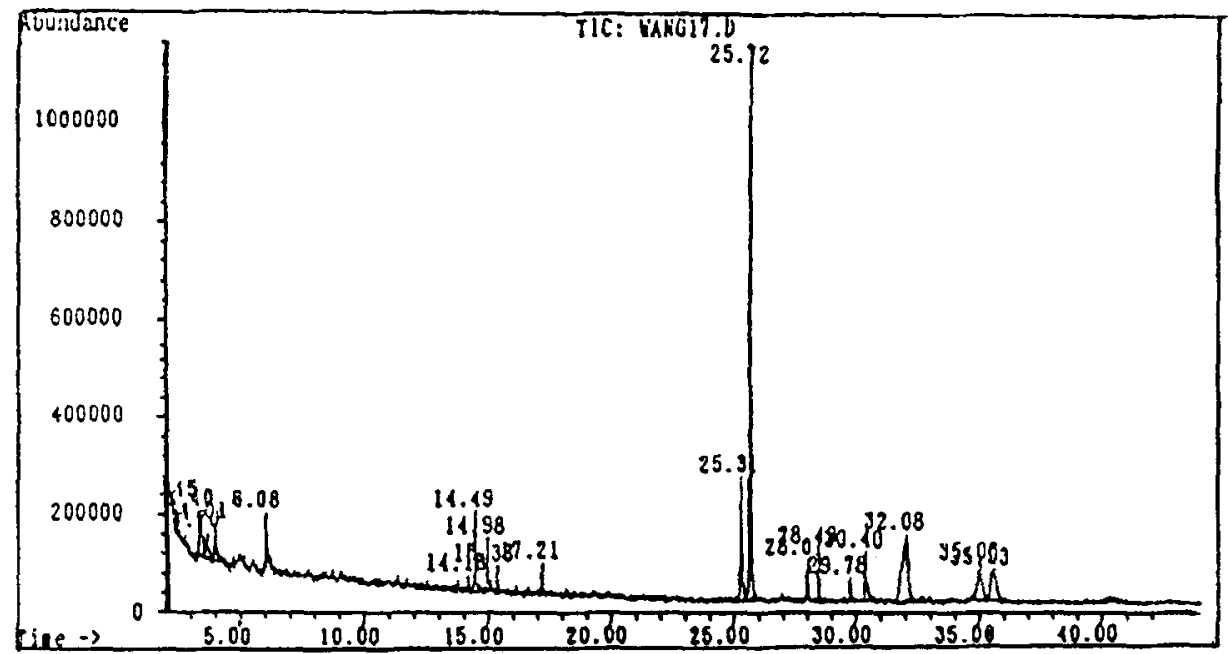

Figure 3. GC chromatogram of the DNPH-derivatives of aldehydes obtained from the Chinese incense smoke.

Figure 2. gives the HPLC chromatogram of the DNPH-derivatives of aldehydes in the smoke collected from a Chinese temple. The above mentioned aldehydes were also found on the chromatogram. The results of GC/MS examination of Chinese incense smoke is shown in Figures 3,4 and 5 . Figure 3 is the GC chromatogram. 

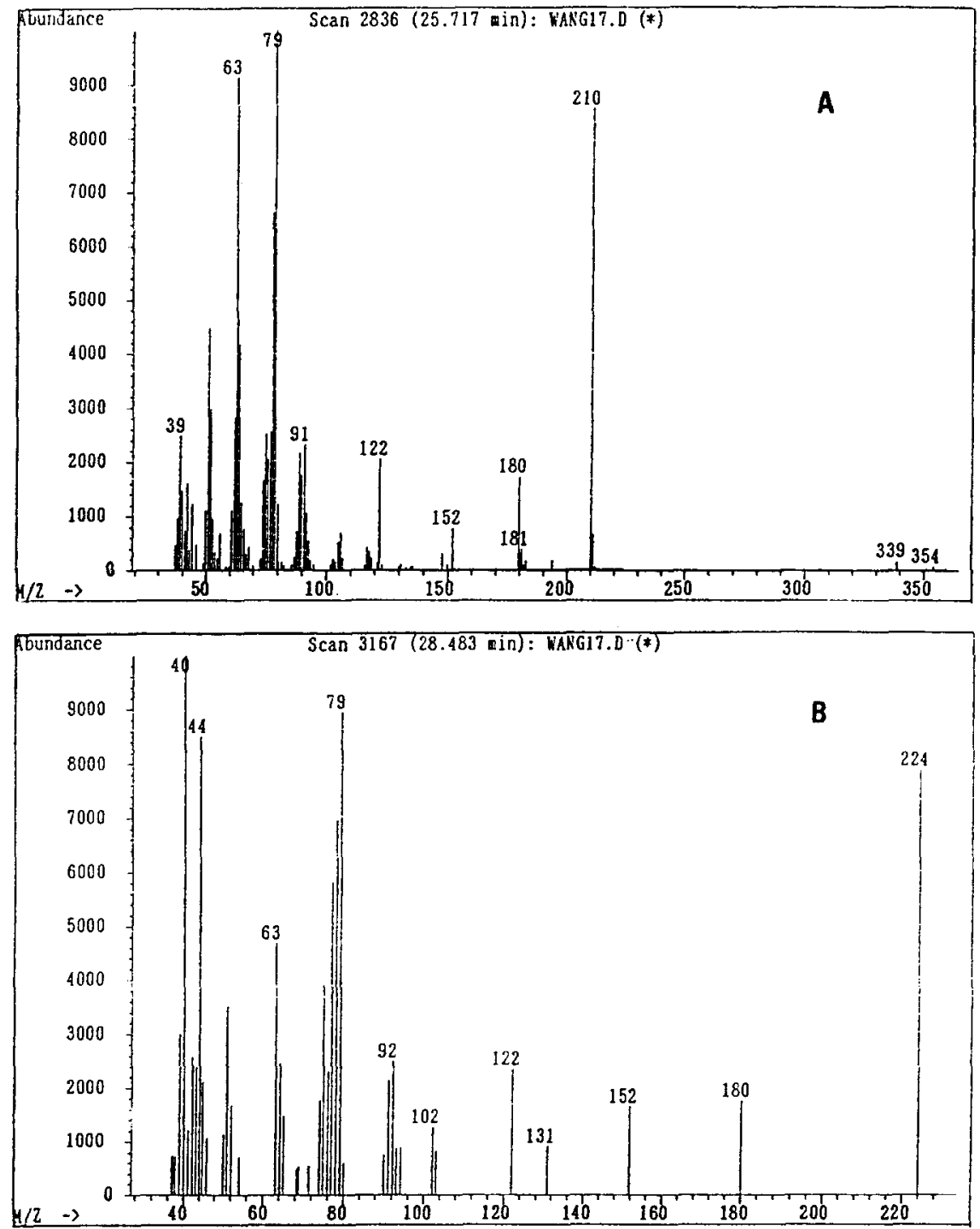

Figure 4. The mass spectra of the DNPH-derivatives of aldehydes obtained from the Chinese incense smoke.

(A: formaldehyde-dini tropheny lhydrazone;

B:ace taldehyde-dini tropheny lhydrazone.)

Figure 4-(A), 4-(B), 5-(A) and 5-(B) show the mass spectra from the compounds with retention time of $25.717 \mathrm{~min}, 28.483 \mathrm{~min}$, $30.406 \mathrm{~min}$ and $32.079 \mathrm{~min}$ respectively. The respective molecular ion pattern at $\mathrm{m} / \mathrm{e} 39-210,40-224,40-236$ and $40-276$ is typical of formaldehyde-DNPH, acetaldehyde-DNPH, acrolein-DNPH and furnanalDNPH. Propionaldehyde was not identified by GC/MS, probably because the amount was so small that it could not be detected. 

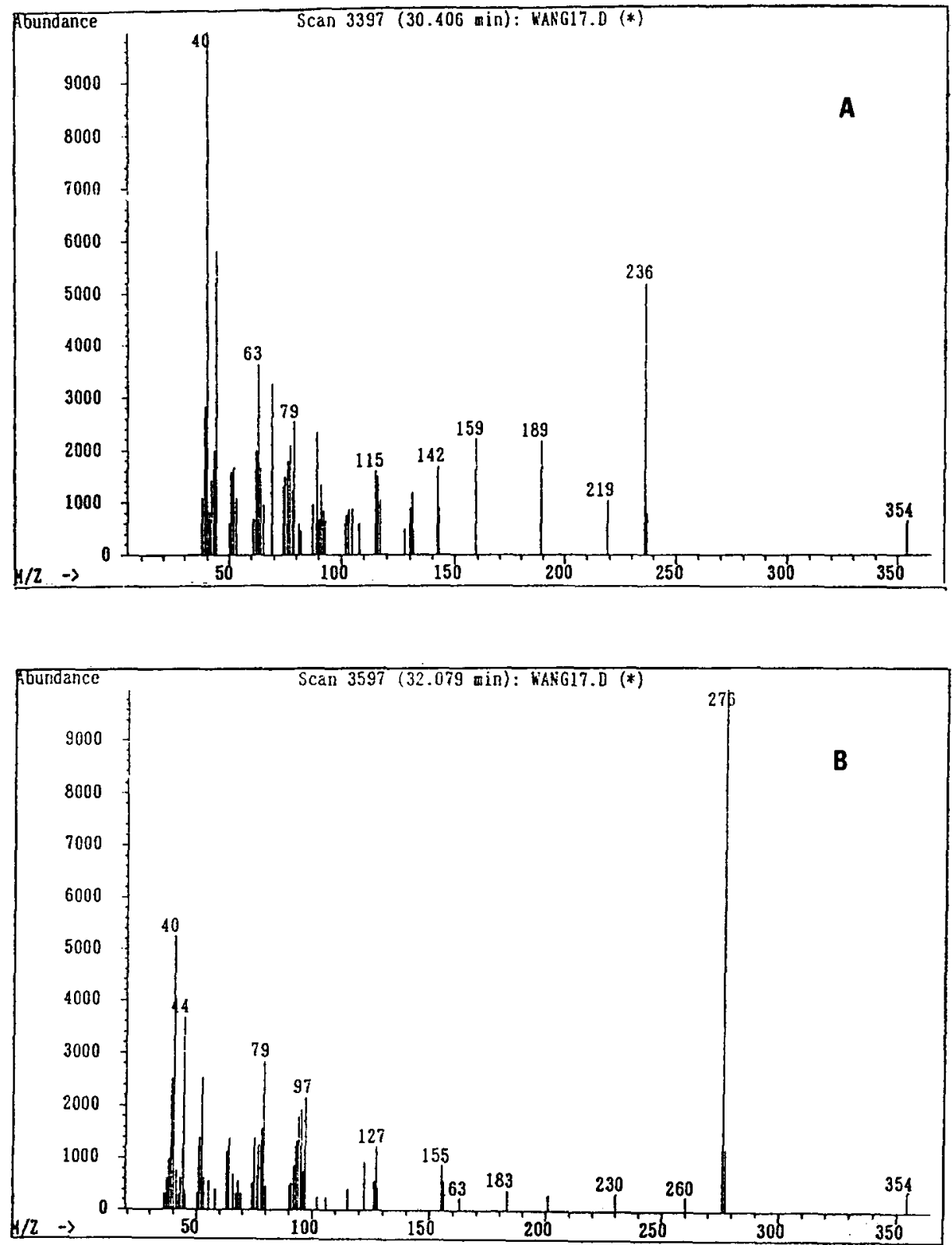

Figure 5. The mass spectra of the DNPH-derivatives of aldehydes obtained from the Chinese incense smoke.

(A:acrolein-dini trophenylhydrazone;

B: furnanal-dini trophenylhydrazone.)

Tables 2. indicates the concentration of each aliphatic aldehyde generated by burning one gram of incense under controlled atmosphere with relative humidity ranging from $46.0 \%$ to $83.2 \%$. The 
result shows that the $\mathrm{ppm} / \mathrm{g}$ varies with the type of Chinese incense and the relative humidity. The concentration of aliphatic aldehydes decreased with an increase of humidity. Overall, formaldehyde was predominant in the smoke from burning Chinese incense.

Tables 2. Generation of aliphatic aldehydes fron burning one gran of incense at various hunidities

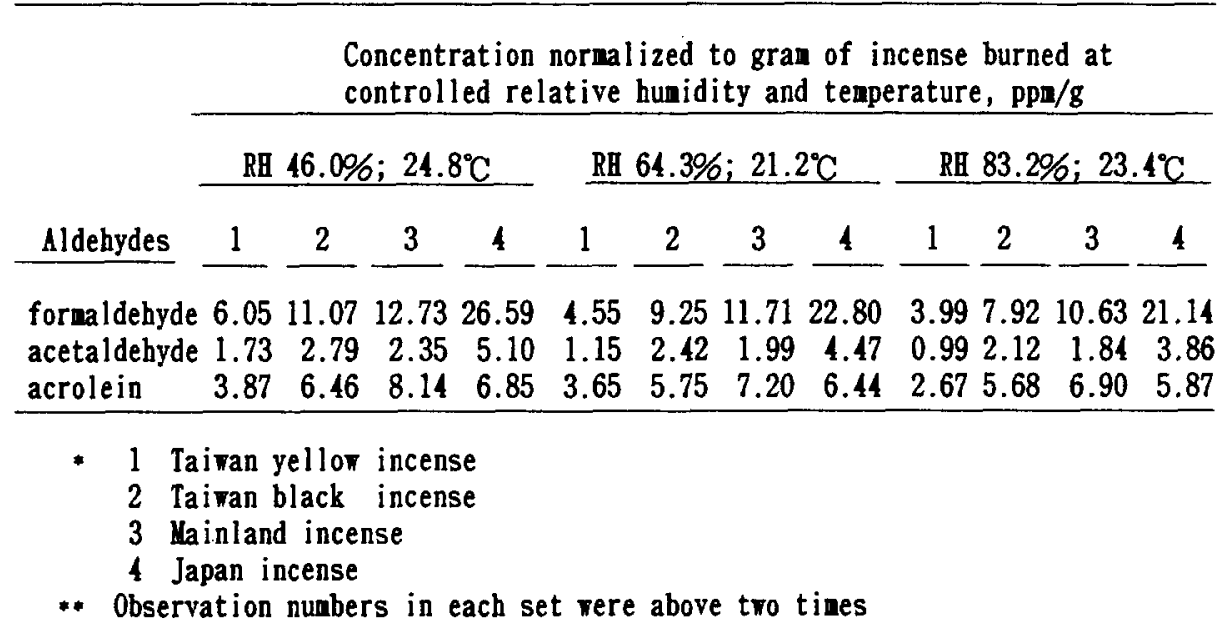

Acknowledgments. This research was funded by a grant from the National Science Council of the Republic of China (NSC-82-0412B002-270). Technical advice was generously provided by $\mathrm{Dr}$. Hui-Po Tang, National Taiwan University and GC/MS was supported by the Center for Industrial Safty \& Health Technology, Industrial Technology Research Institute, Taiwan, the Republic of China.

\section{REFERENCES}

Brandt-Rauf PW, Fallon LF Jr, Tarantini T, Idema C(1988) Health hazards of fire fighters: Exp Assess. 45:606-612

Chen CJ, Tang YF, Shieh T, Chen JY, Liu MY (1987) Multifactorial etiology of nasopharyngeal carcinoma. Head Neck Oncolog Res Conf 10-12:469-476

Crupm DR, Gardiner D(1989)Source and concentrations of aldehydes and ketones in indoor enviroment in the UK. Environ Int 15: 455-462

Grosjean D (1982) Formaldehyde and other carbonyls in Los Angeles ambient air. Environ Sci Technol 16:254-262

Kalabokas P, Carlier P, Fresnet P, Mouvier G, Toupance G (1988) Field studies of aldehyde chemistry in the Paris area. Atoms Environ 22:147-155 
Lin JH, Yao YH (1993) Formaldehyde in conventional homes in Taiwan. Environmental International 19:561-568(in press)

Lowengard RA, Peters JM, Cicioni C, Buckley J, Bernstein L, Preston-Martin S, Rappaport E(1987) Chi ldhood leukemia and parents occupational and home exposures. J Natl Cancer Inst $79(1): 39-46$

Martin SP, Mimi CY, Benton B, Henderson BE (1982) N-nitro compounds and childhood brain tumors $-A$ case-control study. Can Res 42:5240-5245

Mimi CY, Garabran DH, Huan TB, Henderson BE (1990) Occupational and other nondietary risk factors for nasopharyngeal carcinoma in Guangzhou, China. Int J Cancer 45: 1033-1039

Rasmussen RE (1987) Mutagenic activity of incense smoke in sallmonella typhimurium. Bull Environ Contact Toxicol 38: 827 $-833$

Schoenotol R, Gibbard S (1967) Carcinogens in Chinese incense smoke (letter). Nature 216:612

World Health Organization (1987) Air Quality Guidelines for Europe, 91-104 